\title{
An Evaluation of the Literacy Demands of Online Natural Disaster Preparedness Materials for Families
}

\author{
Marvin So, MPH (D); Jessica L. Franks, MPH, CHES; Robyn A. Cree, PhD; Rebecca T. Leeb, PhD
}

\section{ABSTRACT}

Objective: Natural disasters are becoming increasingly common, but it is unclear whether families can comprehend and use available resources to prepare for such emergencies. The objective of this study was to evaluate the literacy demands of risk communication materials on natural disasters for US families with children.

Methods: In January 2018, we assessed 386 online self-directed learning resources related to emergency preparedness for natural disasters using 5 literacy assessment tools. Assessment scores were compared by information source, audience type, and disaster type.

Results: One-in-three websites represented government institutions, and 3/4 were written for a general audience. Nearly 1 -in- 5 websites did not specify a disaster type. Assessment scores suggest a mismatch between the general population's literacy levels and literacy demands of materials in the areas of readability, complexity, suitability, web usability, and overall audience appropriateness. Materials required more years of education beyond the grade level recommended by prominent health organizations. Resources for caregivers of children generally and children with special health care needs possessed lower literacy demands than materials overall, for most assessment tools.

Conclusions: Risk communication and public health agencies could better align the literacy demands of emergency preparedness materials with the literacy capabilities of the general public.

Key Words: children, disaster literacy, emergency preparedness, natural disasters, risk communication

\section{INTRODUCTION}

Natural disasters such as hurricanes, floods, and wildfires are increasing in frequency and intensity, highlighting the need for advanced planning by communities. ${ }^{1,2}$ Although many government, nonprofit, and private actors are involved in emergency preparedness planning, it is the preparedness knowledge and skills of individual families that form the foundation of a prepared community. ${ }^{3}$ The role of families and caregivers has garnered particular interest in the emergency management sector, because they are essential for the prevention of adverse disasterrelated consequences among children who depend on them. ${ }^{4,5}$

Children are often disproportionately impacted by public health emergencies due to their unique physiological, social, and physical characteristics (eg, faster respiratory rate). ${ }^{4,6}$ Further, children with special health care needs $(\mathrm{CSHCN})$ may face additional difficulty in disasters, because they often require specialized equipment, medications, or other considerations distinct from typically developing peers. ${ }^{7}$ In order to make informed decisions about child health and safety, parents and other primary caregivers often turn to the Internet as a resource. ${ }^{8}$ Likewise, community emergency planners and health systems use various risk communication channels to educate families about public health threats and harm mitigation strategies. Ensuring that families are able to understand available resources so that they can take action is paramount, particularly because public health emergencies can overwhelm jurisdictions' standard communications systems. ${ }^{9}$

Recent evaluations demonstrate a mismatch between the literacy demands (ie, cognitive skills necessary to process, understand, and act upon provided content ${ }^{10}$ ) of available materials and the literacy levels of vulnerable populations, such as low-income people of color $^{11}$; pregnant and postpartum women ${ }^{12}$; and people who are deaf or hard-of-hearing. ${ }^{13}$ These populations may not be able to access standard resources offered in emergency preparedness, response, and recovery. They might also face barriers to appropriate action (eg, developing a family communication plan or emergency kit) even when information about hazards is made available. $^{9}$ Recent evidence suggests that caregivers can prepare for emergencies only if they both perceive threat and have the self-efficacy to use available resources to meet situational demands. ${ }^{14}$ Therefore, it is critical for risk communication agencies to pay attention to 
more than the content of disseminated materials. Addressing factors that determine individuals' ability to comprehend and effectively use information, such as literacy demands, might help ameliorate disparities in disaster-related morbidity and mortality.

Unfortunately, evaluation efforts have often only focused on a single dimension of the cognitive demands involved in literacy (eg, readability); not been specific to families or children; or used non-systematic methods to retrieve resources. Further, these assessments typically focus on government resources, even though families draw upon information from a range of trusted sources, including professional (eg, American Academy of Pediatrics) and non-profit organizations. ${ }^{8}$ In recognition of these gaps, we conducted a comprehensive evaluation of the online information environment for personal- or family-level preparedness. Enhanced understanding of the literacy requirements of these sources could guide the development of appropriate messaging prior to crises, help communities tailor risk communication interventions to better reach target audiences, and ultimately keep families safe during and after disasters. ${ }^{9,15}$

\section{METHODS}

We conducted a cross-sectional evaluation of web-based risk communication materials using standard search engines that parents or caregivers in the United States would likely use to search for resources. For the purposes of this study, we defined "parents" or "caregivers" as the person(s) with primary responsibility for the day-to-day care and well-being of the child; this does not include professionals such as educators. We limited our focus to emergency preparedness for natural disasters, given the increasing frequency of these crises.

To address limitations of previous research ${ }^{11,13}$ and a lack of consensus on how to comprehensively assess literacy demands, ${ }^{10,16}$ we used several evaluation instruments. We selected tools informed by 5 dimensions articulated in Brown et al.'s $(2014)^{17}$ disaster literacy framework to assess literacy demands: readability, complexity, suitability, web usability, and overall audience appropriateness. This approach leverages the unique strengths of each tool while offsetting their respective limitations, consistent with similar studies. ${ }^{18}$

\section{Sampling Procedure}

We performed searches on the 3 most frequently used search engines using SimilarWeb.com's (New York, NY) Top Websites Ranking function in January 2018: Google (Mountain View, CA), Bing (Redmond, WA), and Yahoo! (Sunnyvale, CA). These search engines represent more than $95 \%$ of all searches conducted across the globe. ${ }^{18}$ To ensure comprehensiveness, we conducted (1) a general search for emergency preparedness and (2) targeted searches for specific natural disaster event types. We refined our search by

TABLE 1

\section{Search Strings Used for Evaluation of the Literacy Demands of Self-Directed Learning Resources on Emergency Preparedness for US Families}

Concept

General Search

Emergency

Preparedness

Disaster Preparedness

Emergency Readiness

Disaster Readiness

Children's Emergency

Preparedness

Children's Disaster

Preparedness

Targeted Searches

Blizzards

Droughts

Earthquakes

Floods

Heat Waves

Hurricanes

Thunderstorms

Tornadoes

Volcanic Eruptions

Wildfires

\section{Search String ${ }^{a}$}

"Emergency Preparedness"

"Disaster Preparedness"

"Emergency Readiness"

"Disaster Readiness"

"Emergency Preparedness" AND (Child* OR Famil*)

"Disaster Preparedness" AND (Child* OR Famil* $^{*}$

(Blizzard* OR Ice Storm*) OR (Snow Storm*) OR (Winter Storm*)) AND "Preparedness" Drought* AND "Preparedness" (Earthquake* OR Quake* OR Tremor* OR

Temblor*) AND "Preparedness"

Flood* AND "Preparedness"

(Heat Wave*) AND "Preparedness"

(Hurrican* OR Typhoon* OR (Tropical

Storm*)) AND "Preparedness"

(Thunderstorm* OR (Electrical Storm*) OR (Lightning Storm*) OR Thundershower*) AND "Preparedness"

(Tornado* OR Twister* OR Cyclon*) AND

"Preparedness"

(Volcan* OR Erupt*) AND "Preparedness" (Wildfire* OR Brush Fire*) OR (Forest Fire*) AND "Preparedness"

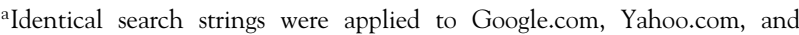
Bing.com.

including terms for children and families and using search strings for the 10 most common natural disasters in the United States. For each string, the disaster type query (eg, "hurricane") was coupled with a query for preparedness (ie, "hurricane" AND "preparedness"), along with common synonyms (Table 1).

\section{Inclusion Screening}

For each search engine, we examined the first 30 results of each search because Internet users infrequently visit websites beyond the 30th result. ${ }^{19}$, a We excluded websites that predominantly or solely contained links to other websites but considered websites linked from these pages for exclusion screening and subsequent coding. This yielded 1430 websites. We did not use search engine optimization features, such as

For the purposes of this study, we assumed that early webpage hits reflected sites that (1) received high quantity of web traffic and (2) members of the general public would be most likely to find and select for preparedness planning. However, ranked search engine results are the result of a more complex set of factors than sole website traffic. Web developers employ search engine optimization (SEO) strategies to improve the web page rankings on search engines (eg, including relevant key words in meta-data, strategically hyperlinking text, using descriptive titles and subtitles on the page). 
TABLE 2

\section{Exclusion Criteria Used for an Evaluation of the Literacy Demands of Self-Directed Learning Resources on Emergency Preparedness for US Families, With Number of Websites Excluded for Each Criterion}

Access Barriers

Broken link

Non-English

Financial cost to access

Login required

Wrong Resource Type

News article

Curriculum

Social media websites

Podcasts/audio/video

All or predominantly links to other pages

Not a self-directed learning

resource

Limited or Wrong Audience

Explicitly non-US

Researcher

Clinician/provider/health care

organization

Prenatal/pregnant only

Government document not

intended for personal/family

preparedness

Retail website

Other Issues

Duplicate

Infographic or PDF that could not

be assessed

Total
Exclusion Reasons

Research article

\section{\# of Websites \% \\ Excluded}

$\begin{array}{cc}26 & 2.5 \\ 0 & 0.0 \\ 2 & 0.2 \\ 5 & 0.5\end{array}$

$30 \quad 2.9$

$32 \quad 3.1$

$\begin{array}{ll}7 & 0.7\end{array}$

$4 \quad 0.4$

$19-1.8$

$138 \quad 13.2$

$110 \quad 10.5$

$4 \quad 0.4$

$5 \quad 0.5$

$0 \quad 0.0$

$82 \quad 7.9$

$10 \quad 1.0$

$558 \quad 53.4$

$6 \quad 0.6$

1044
$6 \quad 0.6$

Assessment of Materials (SAM) instrument ${ }^{23}$ for suitability, (4) National Library of Medicine and National Institute on Aging (NLM/NIA) Web Usability guidelines ${ }^{24}$ for web usability, and (5) Centers for Disease Control and Prevention (CDC) Clear Communication Index (CCI $)^{25}$ for overall audience appropriateness (see Table 3 for details on each tool's scoring range and protocol). For each communication material, we also recorded information source type, audience type, and natural disaster type (see Supplemental Table 1 for protocol).

In certain instances, we needed to make modifications to the assessment tools' criteria, or further operationalize items within tools in order to standardize the coding protocol across coders. We limited such changes to only those that were truly necessary; for example, the SAM includes a criterion for glossy versus matte-based paper, which is not pertinent to viewing resources on screens and was thus not included as an item when calculating scores for that tool. These decisions were applied in a manner consistent with previous literature ${ }^{13,26}$ and are available in Supplemental Table 2.

\section{Literacy Assessment}

MS and JLF independently coded a randomly selected $10 \%$ of websites, and inter-rater reliability was calculated via percent agreement and Scott's $\mathrm{Pi}^{27}$ for every item on each assessment instrument. Scott's Pi was deemed an appropriate measure because it accounts for expected versus observed agreement in the coding of nominal data by 2 coders. These scores were continuously monitored, with coders discussing discrepancies throughout. Before initiating data extraction, scores were deemed "satisfactory" (95\% agreement on average, corresponding to Scott's Pi of 0.85). MS and JLF divided the list of websites in half and coded every included website with each assessment tool using a coding form matrix (available upon request).

settings to match websites to the searcher's geographic region. We excluded advertisements in search results.

Two authors (MS and JLF) divided the list randomly and subjected each website to exclusion criteria within 4 categories (access barriers, wrong resource type, limited or wrong audience, and other issues) informed by previous studies. ${ }^{18,20}$ We focused on free, English-language, self-directed learning resources targeted to the general public in the United States (Table 2 ). We only included websites that received $>1$ visit in the month before the evaluation (based on web traffic data from SimilarWeb.com). This process resulted in 386 unduplicated websites ( $27 \%$ of original sample).

\section{Measures}

Five literacy assessment tools were used to examine distinct domains of literacy demands: (1) Flesch-Kincaid Grade Level ${ }^{21}$ for readability, (2) Peter Mosenthal and Irwin Kirsch (PMOSE/ IKIRSCH) formula ${ }^{22}$ for complexity (3) Suitability
We only considered text that was clearly a component of the page's content, effectively excluding irrelevant text concerning website navigation (eg, "return to top"), advertisements, or embedded social media content. If a website contained gadgets that segmented or hid portions of text to facilitate user comprehension (eg, collapsible modules, section tabs) coders included this content in its entirety, as long as the user was not directed to a new URL when expanding sections. We excluded materials that were solely images without usable text (eg, infographics).

\section{Data Analysis}

We tabulated descriptive statistics and non-parametric tests of association, upon determination of the data set's non-normal distribution using quantile-quantile plots. We tested whether online information literacy assessment scores differed depending on source, targeted audience, and type of natural disaster covered, using Kruskal-Wallis and Wilcoxon rank-sum tests. To account for multiple comparisons, we controlled all tests for false discovery rates using Benjamini and Hochberg's 


\section{There 3}

Constructs Measured and Scoring Protocols Used for 5 Assessment Tools Within an Evaluation of the Literacy Demands of Self-Directed Learning Resources on Emergency Preparedness for US Families

\begin{tabular}{lc}
$\begin{array}{l}\text { Assessment Tool } \\
\text { Flesch-Kincaid Grade Level }\end{array}$ & \multicolumn{1}{c}{ Construct } \\
& $\begin{array}{l}\text { Readability, or years of education } \\
\text { needed (measured by grade-level) } \\
\text { to understand a document based } \\
\text { on sentence and word length. }\end{array}$ \\
& \\
Peter Mosenthal and Irwin Kirsch & Complexity, based on the structure, \\
(PMOSE/iKIRSCH) Formula & density, and dependency of \\
& information in print materials.
\end{tabular}

Suitability Assessment of Materials (SAM)

National Library of Medicine / National Institute on Aging (NLM/ NIA) Web Usability Guidelines

Clear Communication Index (CCI)
Suitability, or how understandable materials are based on how easy it is to comprehend information.

Web usability, or the text readability, information presentation, and ease-of-navigation of websites.
Overall audience appropriateness in consideration of additional dimensions such as clarity and understandability, beyond the basic characteristics that simple readability formulas capture.

\section{Scoring}

Scores range from 0.0 to 12.0

representing school grade year. For

example, a score of 9.3 signifies that the average person who completed the ninth grade would be able to read the material.

Scores range from 1.0 to 5.0 as a sum of the three criteria, and interpreted using a chart to determine complexity level (very low, low, moderate, high, very high); proficiency level (1, 2, 3, 4, or 5); and grade/schooling (range including grade 4 or equivalent to $<8$ years of schooling, grade 8 or equivalent to high school degree, grade 12 or equivalent to some education after high school, 15 years of schooling or equivalent to college degree, or 16 years of schooling or equivalent to post college degree).

The tool rates factors as superior, adequate, or not suitable in six areas (content, literacy demand, graphics, layout and type, learning stimulation and motivation, and cultural appropriateness). Scores range from 0.0 to 100.0 and the total score is interpreted as superior (70-100\%), adequate/below average (40-69\%), or not suitable (039\%).

Scores range from 0.0 to 25.0 , and generate a percentage for the total number of items fulfilled out of a checklist of 24 items. Items capture domains including typeface/weight, text spacing, use of color, simplicity, information presentation, organization, webpage ease-of-navigation, menus, and scrolling.

The score divides the points that the material earned by the total number possible to generate a score that can be interpreted in a binary fashion: excellent (90 or above) or needs work (89 or lower). Items capture domains including main message/call to action, information design, language, state of the science, behavioral recommendations, numbers, and risk.
Doak, Doak, and Root (1996) 23

Hodes and Lindberg (2002) $)^{24}$

Baur and Prue (2014) ${ }^{31}$ procedure. ${ }^{28}$ Differences were deemed significant at $P<0.05$ after these adjustments. We used Stata 14.1 (College Station, TX) for all analyses. Because this was an evaluation of publicly available websites, human subjects' approval was not necessary.

\section{RESULTS}

Assessment tool scores of included websites overall, by audience type, information source, and disaster type are depicted in Table 4.

\section{Website Characteristics}

Of the 386 included websites, government institutions represented the largest group of information sources (119/386, $30.8 \%)$. Private $(98 / 386,25.4 \%)$ and non-profit organizations (81/386, 21.0\%) each comprised more than $1 / 5$ of the overall sample. Less common information source types included news organizations $(41 / 386,10.6 \%)$, educational institutions $(33 / 386,8.5 \%)$, and other sources (eg, individuals' personal 
Assessment Tool Scores for Evaluation of the Literacy Demands of Self-Directed Learning Resources on Emergency Preparedness for US Families, by Information Source, Audience Type, and Disaster Type

\begin{tabular}{|c|c|c|c|c|c|c|}
\hline \multirow[b]{2}{*}{ Characteristics } & \multirow[b]{2}{*}{$N$} & \multicolumn{5}{|c|}{ Literacy Assessment Tool, Mean (SD) } \\
\hline & & Flesch-Kincaid ${ }^{b}$ & PMOSE/IKIRSCH ${ }^{\mathrm{b}}$ & SAM $^{\mathrm{C}}$ & NLM/NIAC & $\operatorname{CDC~CCl}{ }^{c}$ \\
\hline Overall & 386 & $9.6(2.1)$ & $3.5(1.0)$ & $61.6(12.3)$ & $16.9(1.7)$ & $66.6(15.3)$ \\
\hline \multicolumn{7}{|l|}{ Information Source } \\
\hline Government institution & 119 & $9.9(2.3)$ & $3.7(1.1) \dagger$ & $60.5(13.5)$ & $17.1(1.6)$ & $64.3(16.1)$ \\
\hline News organization & 41 & $9.1(2.0)$ & $3.0(1.0)^{* *}$ & $60.2(8.3)$ & $15.5(1.2)^{* *}$ & $64.7(10.4)$ \\
\hline Educational institution & 33 & $9.2(0.8)$ & $3.6(1.2)$ & $62.8(13.2)$ & $16.1(1.4)^{* *}$ & $68.8(12.4)$ \\
\hline Health care institution ${ }^{a}$ & 0 & - & - & - & - & - \\
\hline Non-Profit organization & 81 & $9.5(2.2)$ & $3.6(1.1)$ & $65.0(11.2)^{*}$ & $17.1(1.7)$ & $66.9(16.0)$ \\
\hline Private company & 98 & $9.4(2.0)$ & $3.3(0.6) \dagger$ & $60.5(11.6)$ & 16.8 (1.6) & $69.2(13.5)$ \\
\hline Other & 14 & $8.6(2.1)$ & $3.3(0.5)$ & 59.4 (13.8) & $16.8(2.7)$ & $67.0(20.1)$ \\
\hline \multicolumn{7}{|l|}{ Audience Type } \\
\hline $\begin{array}{l}\text { Parents/caregivers of } \\
\text { CSHCN }\end{array}$ & 18 & $8.3(1.2)^{*}$ & $5.3(2.9)^{* *}$ & $68.7(7.4)^{*}$ & $16.8(2.4)$ & $75.7(10.0)^{*}$ \\
\hline $\begin{array}{l}\text { Parents/caregivers of } \\
\text { children (not CSHCN) }\end{array}$ & 54 & $9.4(2.3)$ & $3.7(0.7)$ & $70.2(10.7)^{* *}$ & $17.9(1.6)^{* *}$ & $73.5(15.5)^{* *}$ \\
\hline Children $^{\mathrm{a}}$ & 0 & - & - & - & - & - \\
\hline General & 272 & $9.5(2.1)$ & $3.4(0.8)$ & 60.2 (11.9) & $16.8(1.5)$ & $65.5(14.8)$ \\
\hline $\begin{array}{l}\text { Other clearly specified } \\
\text { audience }\end{array}$ & 42 & $10.6(2.1)^{* *}$ & $3.5(0.8)$ & $59.2(13.8)$ & $16.1(1.9)^{* *}$ & $63.8(16.8)$ \\
\hline \multicolumn{7}{|l|}{ Disaster Type } \\
\hline Blizzards & 25 & $8.9(1.6)$ & $3.6(1.3)$ & $58.7(13.7)$ & $16.7(1.6)$ & 71.3 (13.7) \\
\hline Droughts & 16 & $10.5(2.0)$ & $3.0(0.0) \dagger$ & $62.9(13.5)$ & $15.5(1.6)^{* *}$ & 67.3 (18.7) \\
\hline Earthquakes & 39 & $8.9(1.6) \dagger$ & $3.5(0.6)$ & $64.2(10.5)$ & $16.7(1.8)$ & 66.1 (12.3) \\
\hline Floods & 33 & $9.5(2.0)$ & $3.9(1.1)^{*}$ & $60.8(10.0)$ & $16.4(2.0)$ & $61.1(21.4) \dagger$ \\
\hline Heat waves & 26 & $8.7(1.4)^{*}$ & $3.4(0.6)$ & 64.7 (11.5) & $17.2(1.4)$ & $64.6(13.7)$ \\
\hline Hurricanes & 31 & $9.5(2.8)$ & $3.5(0.6)$ & $61.6(11.1)$ & $17.5(1.5) \dagger$ & 64.6 (13.8) \\
\hline Thunderstorms & 33 & $8.9(1.4) \dagger$ & $3.3(0.7)$ & $58.4(14.5)$ & $16.8(1.3)$ & $62.0(9.0)$ \\
\hline Tornadoes & 38 & $9.6(1.8)$ & $3.4(0.8)$ & 62.0 (14.5) & $17.0(1.6)$ & $65.4(16.5)$ \\
\hline Volcanic eruptions & 47 & $9.9(1.8)$ & $3.2(0.4) \dagger$ & $62.5(9.7)$ & $16.9(1.8)$ & $68.5(13.8)$ \\
\hline Wildfires & 26 & $9.6(2.0)$ & $3.8(1.1)$ & $62.4(17.6)$ & $16.6(1.7)$ & $68.3(15.9)$ \\
\hline General & 72 & $9.6(1.3)$ & $3.2(0.3)^{*}$ & $63.1(8.8)$ & 16.7 (1.3) & $68.4(10.2)$ \\
\hline
\end{tabular}

Notes: CSHCN: Children with Special Health Care Needs. SD: Standard Deviation. Flesch-Kincaid: Flesch-Kincaid Grade Level, ${ }^{21}$ Range: 0.0-12.0. PMOSE/IKIRSCH: Peter Mosenthal and Irwin Kirsch Document Complexity Level, ${ }^{22}$ Range: 1.0-5.0. SAM: Suitability Assessment of Materials Score, ${ }^{23}$ Range: 0.0-100.0. SAM: Suitability Assessment of Materials Score, ${ }^{23}$ Range: 0.0-100.0. NLM/NIA: National Library of Medicine/National Institute on Aging Web Usability Guidelines Score, ${ }^{24}$ Range: 0.0-25.0. CDC CCI: Centers for Disease Control and Prevention Clear Communication Index Score, ${ }^{25}$ Range: 0.0-100.0.

$* P<0.05, * * P<0.01, \dagger P<0.10$.

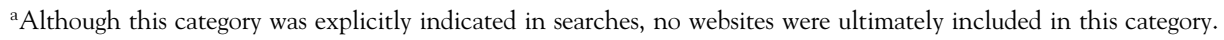

bA lower score for this tool is "better" (ie, has lower literacy demands).

"A higher score for this tool is "better" (ie, has lower literacy demands).

websites; 18/386, 4.7\%). No websites classified as health care institutions were present in the final sample.

Nearly $3 / 4$ of website content did not specify a clear audience or user $(279 / 386,72.3 \%)$. The number of websites targeted to caregivers of children outnumbered those targeted for caregivers of $\mathrm{CSHCN}$ by 3 -fold (54/386 vs $18 / 386$, respectively). There were no websites for which children (ie, individuals $0-18$ years old) themselves were the target audience. Materials that were targeted to neither caregivers nor children, but did specify a clear target audience (eg, childcare providers, business owners), made up $10.8 \%$ of the sample $(42 / 386)$.

Few websites specified a natural disaster type (72/386, 18.7\%). Among those that focused on a specific type of disaster, volcanic eruptions were the most prevalent $(47 / 386,12.2 \%)$ and droughts were least prevalent $(16 / 386,4.1 \%)$.

\section{Website Literacy Demand Scores}

Flesch-Kincaid Grade Level

The mean Flesch-Kincaid score for resources was grade 9.6 (SD: 2.1), indicating that on average, available websites are readable to individuals who have reached the 9 th grade. ${ }^{21, \mathrm{~b}}$ There were no significant differences in Flesch-Kincaid scores across categories of information sources. Materials targeted at parents or caregivers of CSHCN scored at a significantly lower reading level than materials overall $(\bar{x}: 8.3$ grade levels,

In the United States, a 9th grade level corresponds with children who are typically $14-15$ years old. 
$P=0.013)$. Those intended for other clearly specified audiences scored at a significantly higher reading level $(\bar{x}: 10.6$ grade levels, $P=0.005)$. Resources for heat waves were written at a significantly lower reading level $(\bar{x}: 8.7$ grade levels, $P=0.042)$ compared with the average score for all materials in the evaluation. There were no other significant differences by disaster type.

\section{PMOSE/IKIRSCH}

Mean scores on the PMOSE/IKIRSCH complexity measure for included websites was 3.5 (SD: 1.0), indicating a "moderate" degree of complexity and a high school-level of education necessary for a person to use available materials. ${ }^{22} \mathrm{We}$ observed differences in scores by information source, audience type, and disaster type. News organizations evidenced significantly lower complexity scores $(\bar{x}: 3.0, P=0.003)$ compared with materials overall. Materials for caregivers of $\mathrm{CSHCN}$ demonstrated significantly higher scores $(\bar{x}: 5.3, P<0.001)$ compared with materials overall. Materials pertaining to floods also scored higher $(\bar{x}: 3.9, P=0.038)$. Three other disaster type categories (droughts, volcanic eruptions, and general disaster resources) scored lower than the average complexity score for materials overall, but only scores for general resources reached significance $(\bar{x}: 3.2, P=0.016)$.

\section{SAM}

The suitability of materials, as assessed by the SAM, scored 61.6 out of 100 (SD: 12.3) on average. This average score falls into the "adequate or below average" range of scores. ${ }^{23}$ Across information sources, the only source that had a significantly different score than the average were non-profit organizations $(\bar{x}: 65.3, P=0.029)$. When examining audience types, materials for parents of both children with and without special healthcare needs had significantly higher suitability scores than materials overall $(\bar{x}: 70.2, P<0.001$; and $\bar{x}: 68.7$, $P=0.020$ respectively). We found no differences in scores across disaster types.

\section{NLM/NIA Guidelines}

The NLM/NIA guidelines provide an indication of how usable materials are with regard to website characteristics. Overall, materials in this evaluation fulfilled 16.9/25 NLM/NIA guidelines (SD: 1.7). Among the different information sources, news organizations $(\bar{x}: 15.5, P<0.001)$ and educational institutions $(\bar{x}: 16.1, P=0.012)$ fulfilled significantly fewer guidelines. Materials for parents of children (not CSHCN) fulfilled significantly more of the guidelines $(\bar{x}: 17.9, P<0.001)$, whereas those for other clearly specified audiences fulfilled fewer $(\bar{x}: 16.1, P=0.006)$, compared with the average of materials overall. Drought materials were the only disaster type that met significantly fewer guidelines than materials overall $(\bar{x}: 15.5, P=0.002)$.

\section{$\mathrm{CDC} C \mathrm{Cl}$}

The CCI provides a multi-faceted indication of overall audience appropriateness. The mean CCI score we observed for all materials was 66.6 (SD: 15.3), which can be interpreted as "needs work" (as opposed to an "excellent" score of 90 or above) according to the assessment tool. ${ }^{25}$ (Information regarding the CDC CCI score range and domains covered is available in Table 3.) We did not see differences in scores by information source categories. Materials for parents scored significantly higher than all materials on average - inclusive of both parents of CSHCN $(\bar{x}: 73.5, P=0.003)$ and those without special needs $(\bar{x}: 75.7, P=0.017)$. We found no significant differences in scores by disaster type.

\section{DISCUSSION}

Communities that are prepared for emergencies begin with families who are prepared for emergencies. ${ }^{4}$ Increasingly, US families use free information on the Internet to understand children's needs and inform their decisions regarding caregiving concerns, including emergency preparedness. ${ }^{8}$ We evaluated the literacy demands required for caregivers to comprehend the information contained in nearly 400 publicly available online resources. Findings indicate a mismatch between literacy demands and the general public's literacy capabilities, suggesting that families may not have the appropriate information to be adequately prepared for natural disasters. As such, risk communication and public health organizations could consider modifications to available resources to address literacy demands in the areas of readability, complexity, suitability, web usability, and overall audience appropriateness.

\section{Information Sources}

Most available resources were materials issued by government agencies, underscoring the prominent role such institutions play as sources for risk communication to the public. ${ }^{15}$ Notably, less than $1 / 10$ of all resources examined were educational websites. Most children and adolescents spend the majority of their day in schools, which can also serve as family reunification points during emergencies. Thus, educational organizations represent an essential information source in community-level disaster preparedness and response. ${ }^{2,4}$ Our searches also uncovered no resources from health care organizations. This is notable because pediatricians are credible, well-positioned sources for providing anticipatory guidance around emergency preparedness. ${ }^{2}$ Although educational and health care organizations likely possess risk communication materials that were not captured - or that did not otherwise meet inclusion criteria - our evaluation suggests that disseminating messages to families via these trusted sources remains an important gap in the online information environment. ${ }^{7,29}$ Emergency preparedness agencies must engage with educational and health care partners in order to reach the whole community. ${ }^{5,30}$ 


\section{Target Audiences}

Nearly $3 / 4$ of materials in the evaluation did not clearly specify a target audience. This may be a concern, because tailoring messages for intended audiences is a core pillar of risk communication. ${ }^{31}$ We acknowledge that inclusion of search terms tailored to specific audiences might have netted different results; however, this was beyond the scope of our evaluation given our goal to broadly understand literacy demands of materials for families in the United States.

Additionally, our evaluation highlights a lack of resources to help children directly contribute to family-level preparedness planning. Increasingly, researchers and practitioners have advocated for both expanded consideration of children in preparedness and meaningful integration of their perspectives directly into the planning cycle. ${ }^{6,7}$ One promising resource is CDC's Ready Wrigley campaign, which provides emergency preparedness information directly to children through media, including activity books, checklists, and a mobile application (https://www.cdc.gov/phpr/readywrigley/). Evaluating the impacts of Ready Wrigley and similar materials could help make the case for additional resources that speak directly to young people.

\section{Literacy Demands}

For the three assessment tools that offer an ordinal interpretation of continuous scores (PMOSE/IKIRSCH, SAM, CDC $\mathrm{CCI}$ ), materials on average did not meet the optimal category specified for each respective tool. According to both the Flesch-Kincaid readability and the PMOSE/IKIRSCH complexity scores, the average required reading level of assessed materials was higher than the average US adult reading level of 8 th grade. ${ }^{23,32}$ This is also higher than the grade level recommended by prominent health organizations, including the National Institutes of Health ${ }^{33}$ ("Keep within a range of about a 7th or 8th grade reading level") and American Medical Association ${ }^{34}$ ("Write at or below the 6th grade level"). Thus, efforts to improve readability and reduce the complexity of extant materials could augment comprehension and appropriate utilization of these materials. Of note, that different grade levels were interpreted by the Flesch-Kincaid and the PMOSE/IKIRSCH measures highlights the value of employing multiple assessment tools as part of a comprehensive communication planning process. ${ }^{35}$

The suitability of existing resources, measured by the SAM, could also be improved from "adequate/below average" to "superior" through concerted focus on information content, graphics, layout/typography, and learning stimulation. Findings from the NLM/NIA guidelines are difficult to interpret because there is no established recommendation for what constitutes sufficient web usability; nonetheless,

In the United States, 6th grade level corresponds with children who are typically 11-12 years old; 7th grade corresponds with children who are typically 12-13 years old; and 8th grade level corresponds with children who are typically 13-14 years old. materials, on average, complied with $<70 \%$ of the recommendations, suggesting improvements could be made by focusing on domains targeted by this assessment tool (eg, ease-of-navigation, information presentation; see Table 3). For the CCI, materials scored in the 66th percentile, lower than the $90 \%$ criterion that the CDC considers adequately audience-appropriate. Fortunately, many resources are available to risk communication and public health specialists to better align materials' literacy demands with the skill levels of the general public. ${ }^{33,35,36}$

No clear patterns in scores on literacy assessment tools emerged across information sources nor disaster type (see Table 4). For instance, although news organizations had lower complexity according to PMOSE/IKIRSCH (better), they scored lower with regard to web usability based on NLM/ NIA guidelines (worse). When looking at audience type, materials targeted for a particular target audience did appear to fare better than the overall average, compared with those that were not tailored (ie, general audience message). Resources aimed at parents/caregivers of both CSHCN and those without generally had lower literacy demands compared with other materials - both groups demonstrated lower literacy demands than overall averages, for 3 of 5 assessment tools used. This finding is encouraging, because parents may share materials with their children as part of their family emergency planning process. In addition, parents of CSHCN specifically may require additional information to effectively prepare for their children's disaster-related needs. ${ }^{7}$ Relatedly, materials aimed at "other" specified audiences performed better for 2 of 5 tools (Flesch-Kincaid, NLM/NIA). These results are not wholly surprising because several tools contain items that probe directly about target audiences.

Although we are unaware of any evaluation that has examined this scope of websites using this range of tools, a 2008 study did identify that online emergency preparedness resources performed poorer with respect to the Flesch-Kincaid and SAM measures than our estimates for those tools. ${ }^{26}$ This difference suggests that efforts to improve the readability and suitability of public materials may have improved in recent years, though we note the two evaluations are not directly comparable due to study design differences.

\section{Strengths and Limitations}

This study presents findings buoyed by several methodological strengths. With regard to our search strategy, we applied literature from the field of human-computer interaction ${ }^{19,20}$ to develop our approach and used a reproducible protocol within 3 common search engines to capture private and public websites (see Table 2). These strategies increase the likelihood that the materials assessed represent a reasonable approximation of what the public would encounter at the time of the study's writing. We also adjusted for multiple comparisons to minimize the possibility of false-positive findings, augmenting our confidence in 
the differences observed. Further, intentional study design elements, including the use of 2 independent coders, 5 separate tools, and Brown et al.'s framework, ${ }^{17}$ afford us a more comprehensive understanding of extant resources' literacy demands, particularly in the context of this emerging science.

This evaluation is not without limitations. First, we focused solely on natural disasters. Although weather-related events are an increasing cause of morbidity and mortality, ${ }^{1}$ findings may not generalize to other disaster types (eg, human-induced disasters). Second, our search strategy may not have adequately captured the universe of resources that individuals might encounter on the Internet - such as social media and peer forums. ${ }^{8,37}$ Relatedly, conducting searches without localization to specific regions could have affected the websites retrieved in the search (eg, hurricane-related web pages might appear more often if user were to enter the affected region or hurricane name; local school district web pages might not have emerged without explicit mention of one's county). Future work could broaden the scope of materials evaluated, target specific geographic areas, or expand upon our search terms to provide more complete insights. Third, although we sought to characterize the landscape of online resources by making comparisons with average scores of assessed materials overall, we did not make direct statistical comparisons with discrete cutoff points because recommended scores are not available for each tool.

Finally, although the use of multiple tools assessing varied dimensions of literacy enhances the validity of our findings, the comparability and psychometric properties of the tools merit further investigation. ${ }^{10,16}$ For example, 1 study showed that materials revised via the CCI were perceived to be more comprehendible than materials that were not. ${ }^{31}$ However, the $90 \%$ benchmark for classifying a material as "Excellent" (vs "Needs Work") was not developed as a validated cutoff score with an established sensitivity and specificity. Rather, the benchmark was intended as a practical starting point for identifying revisions; the CCI developers recognized that the content of materials is often the product of negotiations between scientists and communicators (C. Baur, e-mail communication, September 2018).

\section{Future Directions}

This study lays the foundation for additional work in this area to better prepare families and communities for natural disasters. When developing or evaluating materials, organizations may consider content-analyzing their materials to illuminate other factors that influence audience comprehension, or to assess other determinants of compliance with preventive behaviors (eg, messaging quality, credibility, accuracy). 19,26 Notably, the CDC's Public Health Emergency Preparedness (PHEP) capability standards were developed to help state, local, tribal, and territorial programs advance preparedness capacity. These standards were recently refined to include elements for vulnerable populations (eg, children, individuals with disabilities). ${ }^{30}$ Assessing materials developed by programs receiving
PHEP support to meet these priorities could help determine whether the needs of children are being effectively integrated for community preparedness and response.

Future efforts might also incorporate the perspectives of families and/or children themselves to elucidate whether findings hold by intended users, using techniques commonly employed within user experience research. ${ }^{13,35,38}$ This would be particularly important because a paucity of literature regarding families' online information-seeking behaviors about preparedness was available to guide our methodology. As others have argued, although guidelines help identify aspects of materials to be improved, their value is diminished without corresponding efforts to test usability with target audiences. ${ }^{12,17}$ Moving forward, communities' actions toward improving the predisaster information environment can be evaluated for their success in effecting behavior change (both in real-time and following actual disasters) using emergent tools (see ${ }^{39,40}$ ).

\section{CONCLUSIONS}

As parents and caregivers continue to turn to the Web for resources on family emergency preparedness, their ability to apply such information when disaster strikes will depend on how understandable the information is. This evaluation offers a global view of English-language risk communication materials that the US general public would likely encounter when conducting Internet-based searches for family-level preparedness as of January 2018. We identified areas that could be addressed to lower the literacy demands of available materials and consequently strengthen their usefulness for families. Achieving national goals of improved health and disaster literacy will require sustained attention to ensuring resources are not only available to the public, but also appropriately matched to their literacy capabilities. ${ }^{36}$

\section{About the Authors}

Centers for Disease Control and Prevention, National Center on Birth Defects and Developmental Disabilities, Atlanta, GA (Mr So, Ms Franks, Dr Cree, Dr Leeb); University of Minnesota Twin Cities, Medical School, Minneapolis, MN ( $\mathrm{Mr}$ So) and Centers for Disease Control and Prevention, Epidemic Intelligence Service, Atlanta, GA (Dr Cree)

Correspondence and reprint requests to Marvin So, University of Minnesota Medical School, 420 Delaware Street SE, Minneapolis, MN 55454 (e-mail: so000012@ umn.edu).

\section{Acknowledgments}

The authors thank Josephine Lau, Melissa Jennings, and Joshua Petimar for their thoughtful guidance in improving the study design and manuscript; and Cynthia Baur for her insights on the Clear Communication Index. Preliminary findings of this research were presented at the 2018 National Association of County and City Health Officials Preparedness Summit (Atlanta, GA). The findings and conclusions in this article are those of the authors and do not necessarily represent the official position of the CDC. 


\section{Funding}

This research was supported in part by an appointment to the Research Participation Program at the Centers for Disease Control and Prevention administered by the Oak Ridge Institute for Science and Education through an interagency agreement between the US Department of Energy and the CDC (MS, JLF).

\section{Conflict of Interest}

The authors have no conflicts of interest to declare.

\section{Author Contributions}

MS and JLF contributed equally to this work.

\section{Supplementary material}

To view supplementary material for this article, please visit https://doi.org/10.1017/dmp.2019.62

\section{REFERENCES}

1. United Nations Office for Disaster Risk Reduction. Global assessment report on disaster risk reduction: making development sustainable: the future of disaster risk management. Geneva, Switzerland; 2015. https:// www.preventionweb.net/english/hyogo/gar/2015/en/gar-pdf/

GAR2015_EN.pdf. Accessed January 3, 2018.

2. American Academy of Pediatrics Disaster Preparedness Advisory Council, Committee on Pediatric Emergency Medicine. Ensuring the health of children in disasters. Pediatrics. 2015;136(5):e1407-e1417. doi: 10.1542/peds. 2015-3112.

3. U.S. Federal Emergency Management Agency. Preparedness in America: research insights to increase individual, organizational, and community action. Washington, DC; 2014. https://www.fema.gov/media-librarydata/1409000888026-1e8abc820153a6c8cde24ce42c16e857/

20140825_Preparedness_in_America_August_2014_Update_508.pdf. Accessed January 24, 2019.

4. Dziuban EJ, Peacock G, Frogel M. A child's health is the public's health: progress and gaps in addressing pediatric needs in public health emergencies. Am J Public Health. 2017;107(S2):S134-S137. doi: 10.2105/AJPH. 2017.303950.

5. Chung S, Gardner AH, Schonfeld DJ, et al. Addressing children's needs in disasters: a regional pediatric tabletop exercise. Disaster Med Public Health Prep. 2018;12(5): 582-586. doi: 10.1017/dmp.2017.137.

6. Peek L, Abramson DM, Cox RS, et al. Children and disasters. In: Rodríguez H, Donner W, Trainor JE, eds. Handbook of Disaster Research. Cham, Switzerland: Springer; 2018:243-262. doi: 10.1007/978-3-31963254-4.

7. Hipper TJ, Davis R, Massey PM, et al. The disaster information needs of families of children with special healthcare needs: a scoping review. Heal Secur. 2018;16(3):178-192. doi: 10.1089/hs.2018.0007.

8. Plantin L, Daneback K. Parenthood, information and support on the internet. A literature review of research on parents and professionals online. BMC Fam Pract. 2009;10(1):34. doi: 10.1186/1471-2296-10-34.

9. Savoia E, Lin L, Viswanath K. Communications in public health emergency preparedness: a systematic review of the literature. Biosecur Bioterror. 2013;11(3):170-184. doi: 10.1089/bsp.2013.0038.

10. Pleasant A, Rudd RE, O'Leary C, et al. Considerations for a new definition of health literacy: discussion paper. Washington, DC: National Academy of Medicine; 2016.

11. James X, Hawkins A, Rowel R. An assessment of the cultural appropriateness of emergency preparedness communication for low income minorities. J Homel Secur Emerg Manag. 2007;4(3). doi: 10.2202/1547-7355.1266.

12. McDonough B, Felter E, Downes A, Trauth J. Communicating public health preparedness information to pregnant and postpartum women: an assessment of Centers for Disease Control and Prevention web pages. Disaster Med Public Health Prep. 2015;9(02):134-137. doi: 10.1017/dmp. 2015.2.

13. Neuhauser L, Ivey SL, Huang D, et al. Availability and readability of emergency preparedness materials for deaf and hard-of-hearing and older adult populations: issues and assessments. PLoS One. 2013;8(2):e55614. doi: 10.1371/journal.pone.0055614.

14. Ryan MT, Rohrbeck CA, Wirtz PW. The importance of self-efficacy in parental emergency preparedness: a moderated mediation model. Disaster Med Public Health Prep. 2018;12(3):345-351. doi: 10.1017/dmp. 2017.80.

15. Abrams MA, Klass P, Dreyer BP. Health literacy and children: recommendations for action. Pediatrics. 2009;124(Suppl 3):S327-S331. doi: 10.1542/ peds.2009-1162I.

16. Kaphingst KA, Kreuter MW, Casey C, et al. Health Literacy Index: development, reliability, and validity of a new tool for evaluating the health literacy demands of health information materials. J Health Commun. 2012;17(Suppl 3):203-221. doi: 10.1080/10810730.2012.712612.

17. Brown LM, Haun JN, Peterson L. A proposed disaster literacy model. Disaster Med Public Health Prep. 2014;8(03):267-275. doi: 10.1017/dmp. 2014.43.

18. Whitten P, Nazione S, Lauckner C. Tools for assessing the quality and accessibility of online health information: initial testing among breast cancer websites. Informatics Heal Soc Care. 2013;38(4):366-381. doi: 10. 3109/17538157.2013.812644.

19. Morahan-Martin JM. How Internet users find, evaluate, and use online health information: a cross-cultural review. CyberPsychology Behav. 2004;7(5):497-510. doi: 10.1089/cpb.2004.7.497.

20. Dhillon G, Coss D, Hackney R. Interpreting the role of disruptive technologies in e-businesses. Logist Inf Manag. 2001;14(1/2):163-171. doi: 10.1108/09576050110363167.

21. Kincaid J, Fishburne RJ, Rogers R, Chissom B. Research Branch Report 8-75: Derivation of New Readability Formulas (Automated Readability Index, Fog Count and Flesch Reading Ease Formula) for Navy Enlisted Personnel. Millington, TN; 1975. http://www.dtic.mil/dtic/tr/fulltext/u2/ a006655.pdf. Accessed March 14, 2018.

22. Mosenthal P, Kirsch I. A new measure for assessing document complexity: the PMOSE/IKIRSCH document readability formula. J Adolesc Adult Lit. 1998;41(8):638-657. doi: 10.2307/40016961.

23. Doak C, Doak L, Root J. Teaching patients with low literacy. JB Lippincott; 1996. https://www.popline.org/node/425267. Accessed March 14, 2018.

24. Hodes RJ, Lindberg DAB. Making your website senior friendly: a checklist. Washington, DC; 2002. https://www.nlm.nih.gov/pubs/checklist.pdf. Accessed March 14, 2018.

25. Centers for Disease Control and Prevention. CDC Clear Communication Index: a tool for developing and assessing CDC Public Communication Products User Guide. Atlanta, GA; 2014. https://www.cdc.gov/ccindex/pdf/clear-communication-user-guide.pdf. Accessed September 21, 2017.

26. Friedman DB, Tanwar M, Richter JVE. Evaluation of online disaster and emergency preparedness resources. Prehosp Disaster Med. 2008;23(5): 438-446.

27. Scott WA. Reliability of content analysis: the case of nominal scale coding. Public Opin Q. 1955;19(3):321. doi: 10.1086/266577.

28. Benjamini Y, Hochberg Y. Controlling the false discovery rate: a practical and powerful approach to multiple testing. J R Stat Soc Ser B. 1995;57: 289-300. doi: 10.2307/2346101.

29. Olympia RP, Rivera R, Heverley S, et al. Natural disasters and mass-casualty events affecting children and families: a description of emergency preparedness and the role of the primary care physician. Clin Pediatr (Phila). 2010;49(7):686-698. doi: 10.1177/0009922810364657.

30. Centers for Disease Control and Prevention. Public Health Emergency Preparedness and Response Capabilities: National Standards for State, Local, Tribal, and Territorial Public Health. Atlanta, GA; 2018. https://www.cdc.gov/cpr/readiness/00_docs/CDC_PreparednesResponse Capabilities_October2018_Final_508.pdf. Accessed December 11, 2018. 
31. Baur C, Prue C. The CDC Clear Communication Index is a new evidence-based tool to prepare and review health information. Health Promot Pract. 2014;15(5):629-637. doi: 10.1177/ 1524839914538969 .

32. National Center for Education Statistics. Adult literacy in America: a first look at the results of The National Adult Literacy Survey. Washington, DC; 2002. https://nces.ed.gov/pubs93/93275.pdf. Accessed August 2, 2018.

33. National Institutes of Health. How to write easy-to-read health materials. Published 2017. https://medlineplus.gov/etr.html. Accessed December 11, 2018.

34. Weiss BD. Health Literacy: A Manual for Clinicians. Chicago, IL: American Medical Association Foundation; 2008.

35. U.S. Department of Health \& Human Services. Quick guide to health literacy. Washington, DC; 2006. https://health.gov/communication/ literacy/quickguide/Quickguide.pdf. Accessed August 2, 2018.
36. National Institutes of Health. Making health communication programs work. Washington, DC; 2013. http://www.cancer.gov/cancertopics/ cancerlibrary/pinkbook/page5. Accessed August 2, 2018.

37. Thomas TL, Schrock C, Friedman DB. Providing health consumers with emergency information: a systematic review of research examining social media use during public crises. J Consum Health Internet. 2016;20(1-2): 19-40. doi: 10.1080/15398285.2016.1142927.

38. Jaspers MWM. A comparison of usability methods for testing interactive health technologies: methodological aspects and empirical evidence. Int J Med Inform. 2009;78(5):340-353. doi: 10.1016/J.IJMEDINF.2008.10.002.

39. Savoia E, Lin L, Gamhewage GM. A conceptual framework for the evaluation of emergency risk communications. Am J Public Health. 2017; 107(S2):S208-S214. doi: 10.2105/AJPH.2017.304040.

40. Bergeron CD, Friedman DB. Developing an evaluation tool for disaster risk messages. Disaster Prev Manag Int J. 2015;24(5):570-582. doi: 10.1108/ DPM-11-2014-0224. 